\title{
Comparison of image restoration methods for lunar epithermal neutron emission mapping
}

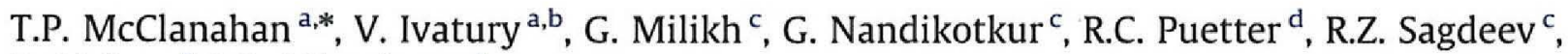 \\ D. Usikov ${ }^{\mathrm{C}}$, I.G. Mitrofanov ${ }^{\mathrm{e}}$ \\ a NASA Goddard Space Flight Center, Astrochemistry Laboratory, Building 34, Room W218, Greenbelt, MD 20771, USA \\ ${ }^{\mathrm{b}}$ Aerospace Engineering Dept, University of Michigan, Ann Arbor, MI, USA \\ 'Space Physics, University of Maryland, College Park, MD 20742, USA \\ d Pixon Imaging LLC, San Diego, CA 92117, USA \\ ' Institute for Space Research, Moscow, Russia
}

\section{A R T I C L E I N F O}

\section{Article history:}

Received 12 June 2009

Received in revised form

16 October 2009

Accepted 30 November 2009

\section{Keywords:}

Geochemistry

Image restoration

Image reconstruction

Neutron

LEND

LRO

Gamma-ray

\begin{abstract}
A B S T R A C T
Orbital measurements of neutrons by the Lunar Exploring Neutron Detector (LEND) onboard the Lunar Reconnaissance Orbiter are being used to quantify the spatial distribution of near surface hydrogen $(\mathrm{H})$. Inferred $\mathrm{H}$ concentration maps have low signal-to-noise ( $\mathrm{SN}$ ) and image restoration (IR) techniques are being studied to enhance results. A single-blind, two-phase study is described in which four teams of researchers independently developed image restoration techniques optimized for LEND data. Synthetic lunar epithermal neutron emission maps were derived from LEND simulations. These data were used as ground truth to determine the relative quantitative performance of the IR methods vs. a default denoising (smoothing) technique. We review and used factors influencing orbital remote sensing of neutrons emitted from the lunar surface to develop a database of synthetic "true" maps for performance evaluation. A prior independent training phase was implemented for each technique to assure methods were optimized before the blind trial. Method performance was determined using several regional root-mean-square error metrics specific to epithermal signals of interest. Results indicate unbiased IR methods realize only small signal gains in most of the tested metrics. This suggests other physically based modeling assumptions are required to produce appreciable signal gains in similar low SN IR applications.
\end{abstract}

Published by Elsevier Ltd.

\section{Introduction}

NASA's Exploration Systems Mission Directorate developed the now orbiting Lunar Reconnaissance Orbiter (LRO) to quantify resources for future human activities on the lunar surface (Chin et al., 2007). The operating Lunar Exploration Neutron Detector (LEND), onboard LRO, was included to provide direct geochemical evidence for water on the Moon (Mitrofanov et al., 2008). However, the efficiency in which neutrons can be detected in lunar orbit is limited by the coupled effects of instrument design constraints and the low lunar neutron fluences at orbital altitudes (Feldman et al., 1993). This results in higher uncertainties or lower signal-to-noise (SN) at the detector which is offset by accumulating samples over a surface region using multiple orbits.

Low SN in geochemical maps has been addressed in similar analytical efforts on data from the precursor Lunar Prospector (1998) mission's neutron spectrometer (LPNS) and gamma-ray

\footnotetext{
* Corresponding author. Tel.: +1 301286 6748; fax: +1 3012860212.

E-mail address: timothy.p.mcclanahan@nasa.gov (T.P. McClanahan).
}

spectrometer (LPGRS) experiments that used image restoration (IR) techniques to deblur and denoise maps (Elphic et al., 2005; Lawrence et al., 2006; Eke, 2001). In this paper we evaluate the potential performance of several IR methods for unbiased restoration of expected lunar neutron emission derived from LEND (Mitrofanov et al., 2008). This includes the results of a single-blind study of IR method performance via direct comparison to synthetic neutron maps and against a default denoising technique designed to quantify signal gains specific to IR deblurring.

While LEND is similar to LPNS in its operations and scientific objectives, there are significant differences in instrument design, expected mapping resolutions and SN that warrant independent evaluation of IR methods. For our single-blind study, four out of twelve invited teams of IR researchers accepted collaboration invitations. Teams configured and submit a total of four IR methods plus a default Gaussian denoising (smoothing) method for evaluation. The blind study was performed in a two-phase process similar to Nesbitt (2004). A preliminary training phase was used to configure and optimize IR methods which provided both "true" and degraded maps. In the subsequent blind study 
phase only "true" maps were provided for restoration (Ivatury and McClanahan, 2009). The primary research objectives are to identify optimal IR methods for LEND map restoration as well as to quantify the expected improvement vs. the default map denoising method. The IR methods and teams include:

1) Iterative Gaussian Smoothing (GSFC-IGS), T. McClanahan, NASA/Goddard Space Flight Center.

2) Regularized Deconvolution (GSFC-UM-RD), V. Ivatury, Univ. of Michigan/GSFC.

3) Weiner (UMD-Fourier), D. Usikov, G. Nandikotkur, G. Milikh, R. Sagdeev, Univ. Maryland (MD).

4) Conjugate Gradient (UMD-CG), Usikov, G. Nandikotkur, G. Milikh, R. Sagdeev, Univ. MD.

5) Pixon (PIXON), Pixon LLC., R. Peutter.

The duration of the paper reviews the necessary background in orbital lunar neutron detection and LEND specific instrument configuration used to formulate a model of the expected epithermal maps. A short description of the IR methods that were selected is provided as well as a description of the protocols that were used for the two-phase blind study. This review also includes the design and formulation of synthetic LEND maps, training, parametric configuration of methods and IR evaluation metrics. The results section quantifies IR method performances and signal gains against the default denoising (Gatissian smoothing) technique.

\section{Background}

The LRO mission was created by NASA in response to the Presidents 2004 Vision for Space Exploration and the tantalizing evidence of hydrogen deposits found at the lunar poles by LPNS (Arnold, 1979; Feldman et al., 1999b; Feldman et al., 2000). LRO successfully entered lunar orbit in June 2009 with scientific objectives that include locating and quantifying volatile deposits, e.g. water, and other potential resources that would support future human exploration of the surface. LEND is measuring lunar surface neutron emissions to refine the spatial distributions and quantity estimates of the putative polar $\mathrm{H}$ deposits identified by LPNS (Mitrofanov et al., 2007).

Hydrogen on the Moon has been postulated for over 30 yeats (Arnold, 1979). Though originally thought to be dry, recent re analysis of Apollo-15 lunar basalts collected at mid-latitudes indicates ambient $\mathrm{H}$ concentration may approach $745 \mathrm{ppm} \sim 0.07 \%$ (Saal et al., 2008). However, LPNS low resolution evaluations of the polar region indicated $\mathrm{H}$ concentrations may approach $\sim 3-5 \%$ and that enhanced concentrations may exist in smaller spots at the poles (Feldman et al., 1998a). It is inferred from these results that $H$ enhanced regions are accumtlated through the combined effects of a regional $\mathrm{H}$ budget driven by variably offsetting factors governing depositional and entrainment processes. H depositional processes include periodic cometary and meteoritic bombardment and/or isotropic influx of solar wind protons (Vondrak and Crider, 2003).

$H$ accumulations can be regionally influenced by various geophysical entrainment effects that constrain the rate of $\mathrm{H}$ sublimation. At extremes some regional sublimation processes may be completely attenuated in polar topographic depressions, where persistent low polar inclination $\sim 1.5^{\circ}$ to the sun produces low and permanent shadowing conditions (PSR)'s with persistently low temperatures $<100^{\circ} \mathrm{K}$ (Neubert et al, 2005; Vasavada et $\mathrm{al}_{\text {, }}$ 1999). These conditions, over time are postulated to minimize $H$ losses from sublimation and assuming isotropic $\mathrm{H}$ influx, may lead to regional $H$ accumulations. As a result, PSR's have been clefined as the primary LEND surface targets (Mitrofanov et al., 2010; McClanahan et al., 2009).

Importantly, the interpretation of orbital neutron measurements only indicates the presence of $H$, not its geochemical form e.g. water $\left(\mathrm{H}_{2} \mathrm{O}\right)$, hydroxyl $\left((\mathrm{OH})^{-1}\right)$ and protons $\left(\mathrm{H}^{+}\right)$. Neutrons are produced in the lunar regolith via isotropic influx of high energy galactic and solar cosmic rays. In this process, subsequent nuclear interactions induce a number of reactions including spallation of neutrons and $\gamma$-ray's in the top few centimeters of regolith. Neutrons propagate through the regolith interacting with atoms, thereby thermalizing (losing energy) until reaching thermal equilibrium and finally being absorbed in the regolith, or importantly, lost into space as a leakage flux. Rates at which neutrons thermalize (lose energy) in materials are dependent on the material density and atomic cross section of atoms encountered during this process. Compared to most elemental constituents of planetary materials, $H$ has a high cross section to neutrons. As a result, the energy distribution of the neutron leakage flux reflects intensity deficits at epithermal energies commensurate with higher $\mathrm{H}$ concentrations (Feldman et al., 1993). Lunar $\mathrm{H}$ concentrations are derived from detected neutron distributions cross referenced to radiation and neutron transport modeling that factors regolith elemental compositions with variable $H_{1}$ neutron energies and detector/geometric configurations, e.g. Monte Carlo N-Particle Transport (MCNPX) codes (Pelowitz, 2008).

The areal scale of most polar PSR targets is small $<$ few $\mathrm{km}$ diameter and the LPNS uncollimated field of view (FOV) at low altitude is inferred to be larger $(\sim 44 \mathrm{~km}$ FWHM) than the diameter of many PSR's (Feldman et al., 1999a; Bussey et al, 2003). From the areal disparity in the expected small area of PSR's and the larger LPNS orbital FOV, it is inferred some localized hydrogen deposits may exceed LPNS estimates due to the effective averaging (blurring) of variable epithermal tate regions within the FOV. LEND's design enhances its spatial resolution using a passive neutron collimator containing neutron absorbing materials, ${ }^{10} \mathrm{~B}$ and polyethylene, to effectively discriminate surface emissions of epithermal neutrons to within $\pm 5.6^{\circ}$ of the instrument boresite. From its nominal $50 \mathrm{~km}$ mapping altitude, this configuration yields an instrument FoV that subtends a $\sim 10 \mathrm{~km}$ diameter surface region (footprint). LEND will primarily point nadir and integrate signals at $1 \mathrm{~Hz}$ rates using its 10 internal and external detectors. These detectors monitor the energy spectrum of low (thermal), medium (epithermal) and high (fast) energy neutrons emitted from the lunar surface and from the spacecraft. To increase $S N$, four identical co-aligned nadir pointing epithermal neutron detectors are implemented in the collimator, as well as a total of six detectors positioned intemal and external to the detector system for tracking background, instrument and spacecraft induced neutron fluxes (Mitrofanov et al., 2008, 2009).

\subsection{Image and epithermal neutron map reconstruction}

Image formation is a stochastic process that convolves a true signal $f$ degraded by instrumental or environmental blurring $w$. with additive noise functions $\mathbf{n}$, to produce an output image $\mathbf{g}$. where $\mathbf{g}=\mathbf{f} \otimes \mathbf{w}+\mathbf{n}$ (Peutter et al, 2005; Gonzalez and Woods, 1993). Instrumental blurring processes degrade the original image $f$ as a convolution $\otimes$ with the instrument PSF, $w$ and statistical counting noise is added, $\mathbf{n}$ (Poisson). Image restoration techniques aim to reverse the blurring process $\mathbf{w}^{-1-t}$ and attenuate the noise $\mathbf{n}_{\text {. }}$ to obtain an estimate of the original image, $g^{\prime} \approx \mathbf{f}$. In high SN applications many types of image restoration method have been shown to yield significantly improved results (Peutter et al., 2005). However, image restoration becomes increasingly proble matic with lower $\mathrm{SN}$ applications due to the combined effects of 
statistical noise being variably spatially correlated. In these conditions spatial frequencies may intersect with real image frequencies and increase the difficulty in estimating an underlying image formation model. Additionally, some restoration processes may amplify noise signals, yielding artifacts e.g. fluctuations (mottling), which strongly resemble real signals (McClanahan et al., 2007; Ugbeme et al., 2007).

Accurate map formation models for orbital neutron remote sensing are also difficult to estimate due to the low signal rates and variances in environmental and instrumental sampling conditions inherent in long duration mapping. Efforts to mitigate these issues and to optimize reconstructions include technisues to extend map formation models by use of other physically based constraints. However, the implementation of physical constraints in the IR process is performed through either parametric biases or to establish prior physical limits for some pixel values (Elphic et al. 2007; Eke et al., 2009). Eke et al. claim signal gains in restoring LPNS data only with the implementation of physically based constraints, e.g. shadowing. Concerns with this approach are that the lunar polar hydrogen budget is presently poorly understood. Implementing these assumptions as j'estoration priors may neglect either the impact or appropriate weight of other geophysical processes. Results of non-biased IR techniques on LPNS data were equivalent to the same data smoothed with a Gaussian operator, underscoring the importance of accurate priors and understanding the ramifications of potential bias errors in low SN IR applications (No Free Lunch) (Wolpert and Macready, 1997).

Fig. I illustrates LEND's neutron map formation process including required functions and parameters to configure the described IR methods. This includes surface emission and instrumental processes e.g. PSF $\boldsymbol{w}$, background estimation and techniques used to map neutron detections from orbit. Poisson counting statistics, $\mathbf{n}$ describe the arrival process of neutrons accumulated by the detector over a given region and defines the expected pixel uncertainty as sqrt(n). A consequence of this long term multisample mapping strategy for IR e.g. LPNS, is that as opposed to traditional higher SN IR models, each detector measurement accumulated to the map is uniquely a function of time, instrument operations, position and background. As a result, new variables are introduced to the map model e.g. altitude, solar flux, position, pointing which impact estimates of the PSF and statistical uncertainties needed for image reconstruction. For instance, altitude variance in combined measurements at a given pixel $i$,

\section{LEND collimated ${ }^{3} \mathrm{He}$ sensor}

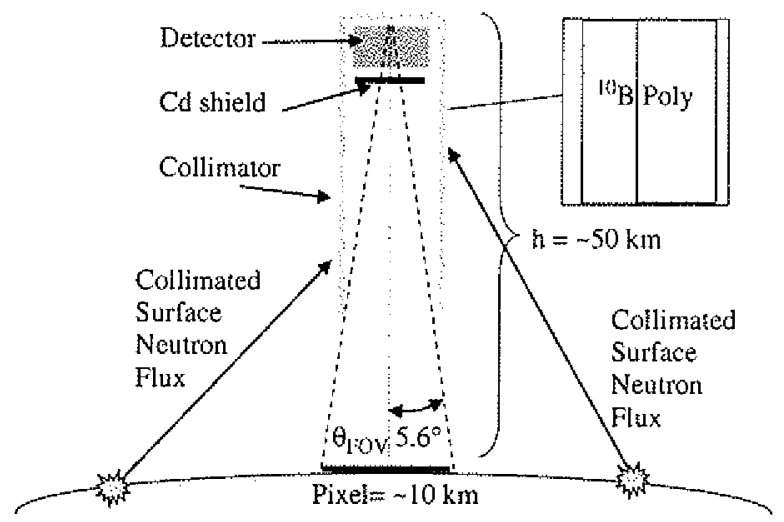

Fig. 1. A single LEND ${ }^{3} \mathrm{He}$ collimated detector configuration and orbital factors defining a nadir pointing collimation process, lunar surface flux of neutrons incident the collimator exterior are passively discriminated using neutron absorbing materials. Collimation yiełds a pixeł size $\sim 10 \mathrm{~km}$ from $50 \mathrm{~km}$ altitude $(h)$ : adversely impacts both the instrument blurring function $w$ and statistical uncertainty $\mathbf{n}$ estimates at $i$. As a result, accumulation of neutrons from variable sampling conditions to the map incurs additional systematic errors.

\section{Methods}

The IR performance study was implemented as a two-phase process using four independent research groups, Section 1. GSFC-IGS is defined as the benchmark "denoising only" approach and establishes the null hypothesis. Each IR method uses different numbers and types of parameters which must be configured for a given application domain. To configure and optimize these methods for LEND map restorations, a preliminary set of 12 synthetic LEND epithermal maps were provided, simulating north, south, polar (NP, SP) and randomized PSR spatial and intensity distributions. Training phase data was distributed to teams and included both degraded synthetic LEND maps $g$ and ground truth maps $\mathbf{f}$. Maps for both the training and blind study phases were produced using LRO mission planning ephemeris for the 382 day primary exploration mission with the assumption of continuous nadir pointing of the LEND instrument and $100 \%$ duty cycle. This virtual flight process effectively flew the LEND instrument mission over quasi-randomly derived lunar polar neutron emission models ( \pm ) 80-90 factoring, ephemeris, 1 second integrations and LEND's expected PSF (Mitrofanov et al., 2009).

A consequence of LEND's collimated design is that the exact surface emission points of detected neutrons are only statistically defined within the FOV. This spatial uncertainty constitutes the present basis of LEND's symmetric, 2-D normal PSF, $w, N(\mu=0$, $\sigma_{x y}=1.67 \mathrm{~km}$ ) from $50 \mathrm{~km}$ altitude. Where, $w$ defines the spatial probability distribution of subtended pixels. The PSF is defined theoretically via the $5.6^{\circ}$ aperture and collimator length, assuming complete discrimination of incoming epithermal neutrons incident to the external side of the collimator. Only epithermal neutron detection processes are assumed with statistical counting uncertainties and no degradation due to motion blur. The map formation process for training and blind studies is illustrated in the flow diagram, Fig. 2. Examples of the LEND map model components are illustrated in Figs. 1 and 3, where the LEND 382 day coverage

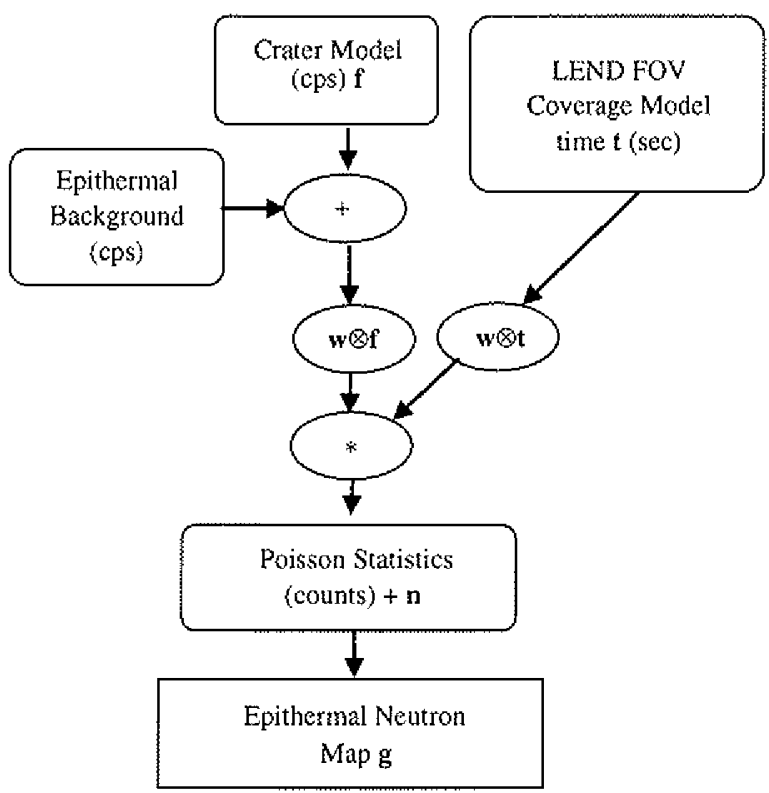

Fig. 2. Flow diagram for generating synthetic lunar epithermal maps. 
distribution over the NP region is depicted in Fig. 3a. A synthetic true lunar epithermal map with craters as depressions in the epithermal count rate (dark spots) $\mathbf{f}_{\mathbf{r}}$ is defined in Fig. 3b. Craters delimit sources of enhanced $(H)$ that have lower epithermal flux rates than surrounding background. These regions deviate randomly up to $18 \%$ below the expected $0.88 \mathrm{cps}$ collimated rate which is equivalent to the $4,5 \%$ suppression seen by LPNS at the lunar north pole. (Feldman et al., 1998b). Crater locations, sizes and intensities were defined in (40) maps randomly, and in known (40) south polar and (40) north polar craters defined by International Astrophysical Union (IAU) conventions (Anderson and Whitaker 1982; Bussey and Spudis, 2004).

Blurring of the image in Fig. 2 is performed and the product of the blurred time and blurred true image $(t \otimes w) *(f \otimes w)$ provides the counts map upon which random Poisson statistical uncertainties $\mathbf{n}$ are added. Factors for randomly deriving the synthetic neutron epithermal maps used in training and single-blind study are listed in Table 1.

\subsection{Map restoration methods: iterative Gaussian smoothing (GSFC-IGS)}

Gaussian smoothing (GS) has significant precedence in signal denoising applications and has been used to smooth published planetary neutron and gamma-ray geochemistry maps
(Mitrofanov et al., 2002; Boynton et al,, 2004). Iterative Gatussian Smoothing (IGS) has been shown to be an equivalent process to a diffusion process (Koenderink, 1984: Perona and Malik, 1987). This denoising method systematically smooths data by incremen tally attenuating the power in high frequency signats first and progressing towards attenuating the power in lower spatial frequencies Costa and Cesar (2001). In the GSFC-IGS approach, the detected signal, $g$ is iteratively smoothed $j$ times. For each iteration the map $\mathbf{g}_{i}$ is convolved with a fixed size, unit volume

Table 1

Synthetic neutron enission map generation factors for training and blind study.

\begin{tabular}{|c|c|}
\hline Map size & $|620 \times 620|$ pixels, pixels $1 \mathrm{~km}^{2}$ \\
\hline Map evaluarion area & $\begin{array}{l} \pm 80-90^{\circ}, \text { pole pt }[310,310]+303 \mathrm{~km} \\
\text { radius }\end{array}$ \\
\hline Predefined North Craters ( 40 ) & 85 (size, position fixed per crater ID) \\
\hline Predefined South Craters (40) & 129 (size, position fixed per crater lD) \\
\hline Predefined Random Craters (40) & Random Size, Random Position \\
\hline Crater intensities & $\begin{array}{l}{[3,6,9,12,15,18] \% \text { decrease }} \\
\text { (random/crater) }\end{array}$ \\
\hline $\begin{array}{l}\text { Accumulated signal scale/382 day } \\
\text { baseline }\end{array}$ & $\begin{array}{l}(1.0,1.75,2.5) \times \text { Accum, cover } \\
\text { (random/crater) }\end{array}$ \\
\hline $\begin{array}{l}\text { Background intensity (blobby, variable } \\
\text { smooth) }\end{array}$ & Mean $=0.88 \mathrm{cps}$, range $15 \%$ of mean \\
\hline Point spread function (field of view) & $N\left(j=0, \sigma_{x, y}=1.67\right)$ \\
\hline Ephemeris 382 days & LRO. Beckman-V4, 2007 \\
\hline Spacecraft polar altitude varjable & $(30-60) \mathrm{km}$, mean $=50 \mathrm{~km}$ \\
\hline
\end{tabular}

a

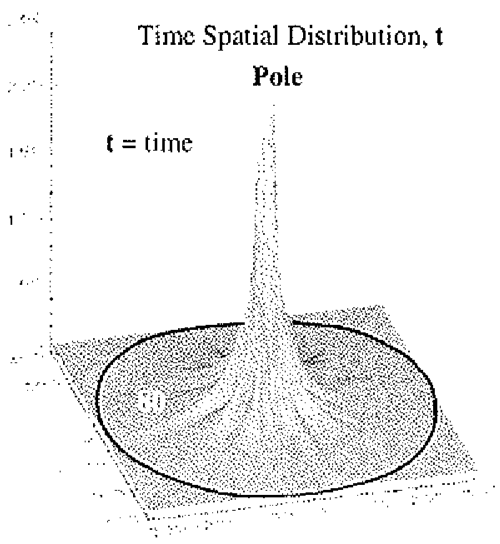

b

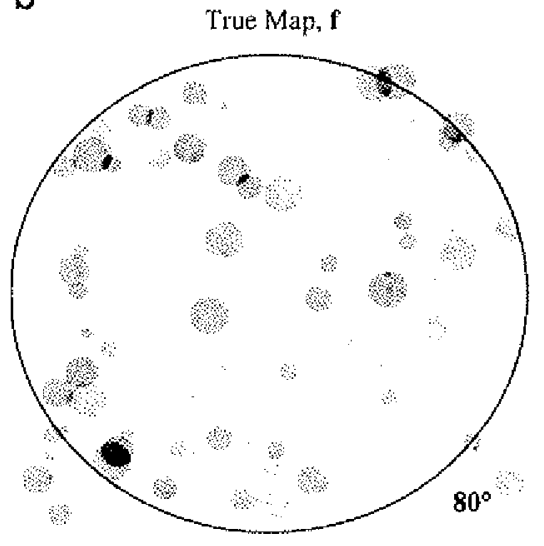

Blurred, Noisy

Count Rates, cps
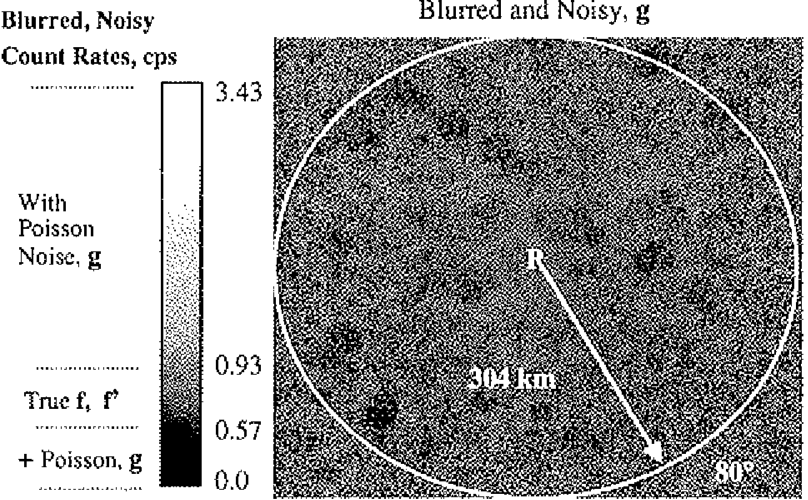

d

True, Deconvolved Count Rates, cps

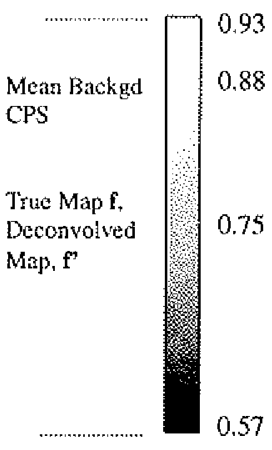

Deconvolved Map, $\mathbf{f}$

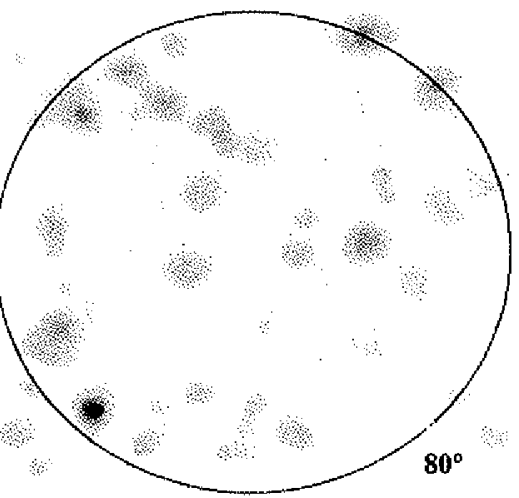

Fig. 3. (a) (Top left) LEND iunar pole accumulated spatial coverage ( $t=$ time) distribution $80-90^{\circ}$ for 382 days ephemeris using $1 \mathrm{~km}{ }^{2}$ pixels. Simulation projects the LEND FOV in $1 \mathrm{~s}$ integrations to a base map. Double lobes in the distribution are due to lunar gravitational anomalies impacting LRO trajectories near the pole (P). (b) (Bottom left) Example true cotnt rate map fit, time normalized with randomized placement and crater intensity (dark spots, variable intensity) blobby background, $\mu=0.88$ cps, range $< \pm 7.5 \%$. Range $=[0.57,0.93]$ cps. Craters represent randomly defined, discrete decreases in epithermal count rates, $\left[3-18 \mid \mathbf{z}^{2}\right.$. Area $>80^{\circ}$ latitude evaluated by methods = 288413 pixels/map. (c) (Top right) Example time normalized blucred and noisy map g/t , after convolution of true map (b) with LEND FOV and added Poisson statistical uncertainties from map used as input to IR methods. Range $=[0 ., 3.43$ l cps. Metrics compare reconstructed maps against the true map (b). (d) (Bottom right) Example of LEND epithermal map after image restoration (PIXON), $f^{\prime} / \mathrm{t}$, Range $=[0.57,0.93$ ]. 
2-D Gaussian smoothing kernel $\mathbf{k}_{1} \mathbf{g}_{j+1}=\mathbf{g}_{\mathbf{j}} \otimes \mathbf{k}$. For each true training map $f$, the root-mean-square (RMS) error is quantified at each iteration $j$ comparing true $\mathrm{f}$ and smoothed $\mathrm{g}_{j}$. For each training map $i$, the minimal RMS error is determined after each iteration. An average of the IGS RMS error training curves yields [11 iterations] as the optimal iteration setting $j$ to denoise the blind study dataset (GSFC-IGS).

\subsubsection{Regularized IR: (GSFC-UM-RD)}

The GSFC-UM-RD method optimizes a regularized deconvolution function (DECONVREG) available via the MATLAB ${ }^{\text {it }}$ signal processing package. A regularization strategy minimizes the degree of noise amplification incurred from division of small $\rightarrow$ 0 , high frequency Fourier coefficients common in Weiner type IR methods (Weiner, 1942), In this approach, inverse filtering is performed using a variant of the least squares method, $\Sigma[\mathbf{g} \cdots \mathbf{W} \otimes \mathbf{f}]^{2}+\alpha[\mathbf{p} \otimes \mathbf{f}]^{2}$. Regularization term $\mathbf{p}$ defines a discrete Laplacian distribution convolved with $f$ yielding a smoothed function scaled by $\alpha$ (LRANGE), defining a spatially specific noise minimization constraint. A second parameter in the function is known as the additive noise power, $n p$ of the image. $n p$ is calculated using a 2-D Fast Fourier Transform (FFT) of g. A center inverse fourier transform is then applied to the blurred image $\mathrm{g}$. Optimal settings for $N P, L R A N G E$ parameters were defined via gridded search of the discretized DECONVREG input parameter space. Optimal input parameter settings [32, 993].

\subsubsection{Weiner (UMD-Fourier)}

A fast Fourier transform (FFT) based method, developed by (Press et al., 2008; Weiner, 1942) is well known for speed, and has application to signals corrupted by noise and coordinate-jnvariant point spread function $\mathbf{w}$ (Section 3.1). The simplicity of the method lies in the fact that the FFT of the detected image is the product of FFT's of the blurring function $w$ and true image $f$, $F F T(\mathbf{g})=F F T(\mathbf{w}) * F F T(\mathbf{f})$ thereby enabling inversion $F F T(\mathbf{f})=F F T(\mathbf{g}) /$ $F F T(\mathbf{w})$, yielding an estimate of the true image (FFT ${ }^{-1}$ ), $\mathbf{f}^{\prime}$. However, the inversion becomes problematic in the presence of noise (low $S N$ ), and small high frequency coefficients in the denominator that can amplify noise. We address the issue of small FFT coefficient division using additional frequency dependent terms in the denominator $[a, b]$ (a variety of the Wiener filter) as $\mathrm{FFT}(g) /\left[\mathrm{FFT}(\mathrm{w})+a+b * \mathrm{FFT}\left(\mathbf{f}^{\prime \prime}\right)\right] . \mathbf{f}^{\prime}$ is an estimate of the true image. This approach optimizes model parameters using the trial images via gridded search of the input parameter space and obtained the following settings [1e-04, 1e-05] (Rosenfeld and Kak, 1982).

\subsubsection{Conjugate Gradient (UMD CG)}

Performance of the UMD-Fourier method is dependent on event statistics in the individual pixels. These problems are treated using a Conjugate-Gradient method (CG) (Nguyen et al., 2001). Reconstruction is performed by minimizing the leastsquare-method (LSM) sum, $\mathbf{S}=\frac{1}{2}\left(\boldsymbol{w}^{\mathrm{T}} \mathbf{f}-\mathbf{g}\right)^{\mathrm{T}} \Sigma^{-1}\left(\boldsymbol{w}^{\mathrm{T}} \mathbf{f}-\mathbf{g}\right)$ which translates to solving the matrix equation $A f=w^{T} g$, where the $A=\mathbf{w}^{\top} \sum^{-1} \mathbf{w}$, and terms $\mathbf{w}, \sum$, $f$ and $g$ are the point spread function, the covariance matrix of event statistics (in our case-a diagonal one), the true(unknown) and convolved images in vectorized form. Although the solution is a simple matrix inversion, its computational complexity, $O\left(620^{2}\right)^{2}$ precludes processing via conventional computer hardware.

Alternatively, gradient descent methods such as $\mathrm{CG}$ can mitigate the inversion limitation by iteratively adjusting method parameters via discrete estimation of parametric gradients. Methods converge in a limited number of iterations assuming initial parametric estimates are proximal to the global solution. For low SN, a regularization term is included, e.g. $A=w^{T} \sum^{-1} w+$ $\gamma L$, where $\gamma$ is a positive regularization coefficient for noise suppression, $\mathbf{L}$ is a Laplacian circular distribution. The Least Square Method (LSM) sum becomes $S=\frac{1}{2}\left(\mathbf{w}^{\top} \mathbf{f}-\mathbf{g}\right)^{\mathrm{T}} \Sigma^{-1}\left(\mathbf{w}^{\mathrm{T}} \mathbf{f}-\right.$ g) $+\gamma$ Lt. Simulations with the trial images yielded optimal reconstructions using [600 iterations, $\gamma=1000$ ].

\subsubsection{Pixon LLC (PIXON)}

The Pixon ${ }^{2}$ method is a picture-information-based method that performs image reconstruction under the constraint that the model for the image should be the simplest model consistent with the data (Puetter et al., 1995, 2005). PIXON gains its power by imposing a minimum complexity constraint on the reconstructed image. Unlike many other competing technigues, the minimum complexity constraint strongly suppresses unwanted image artifacts, as such artifacts are unneeded in fitting the data, and hence are by definition removed. PIXON proceeds by localized smoothing of a minimized set of independent map sub-regions (Pixon elements). Formally, this is described as an integral over the map. $\mathbf{I}(\mathbf{y})=\int d z K(\mathbf{y}, \mathbf{z}) \varphi(\mathbf{z})$, where $\mathbf{I}$ is a pseudo-image, $\mathbf{K}$ is a positive valued kemel function, $\varphi$ is a spatial scale adaptive regularizing term sensitive to localized noise estimates and limits high frequency fluctuations at scales $<\mathrm{K}$. PIXON regions are dynamically size adaptive as a function of local uncertainties. Convergence to a solution comprises a parallel optimization seeking largest kernel functions $\mathbf{K}$ and minimal least squares fit of the image data for each pixon.

There are several options for implementation and configuring PIXON parameters. For this research INIT_ANNEAL and ANNEAL parameters were optimized in the training process using a gridded search of the 2-D parameter space $[5 \mathrm{e}-6,6 \mathrm{e}-4]$. Maps were preprocessed prior to PIXON processing using a spatially adaptive flux preserving filter to identify regionally variable $\mathrm{SN}$ for an optimally smoothed result.

\section{Results: RMS error $\pm \mathbf{8 0 - 9 0 ^ { \circ }}$}

The main goals of this research are to evaluate and quantify the denoising and deblurring properties of IR methods (Section 3.1) on expected LEND epithermal neutron maps. This includes (1) identify IR methods applicable to LEND (2) theit parametric configuration (3) potential for induction of artifacts and (4) quantify the error reduction specific to IR Vs. the default GSFC-IGS denoising approach. Metrics for method evaluation entail comparing the database of 120 restored maps $f^{\prime}$ against the known synthetic maps $f$, by averaging the RMS error of map $i$ regions/signals of interest reg, with pixels in regions $j$, Eq. (1). Secondary metrics include analysis of residual error distributions and pixels quantified as statistical outliers

Region_RMSError $=\frac{1}{120} \sum_{i=1}^{120} \sqrt{\frac{1}{n} \sum_{j=1}^{n}\left(\mathbf{f}_{i, \text { reg }}(j)-\mathbf{f}_{i, r e g}(j)\right)^{2}}$.

Table 2 states each methods mean RMS error results averaged over the 120 blind study maps. Columns indicate region specific evaluations: (1) Global RMS error for $\pm 80-90^{\circ}$ region followed by the decomposition of each map $i$ into (2) Background, (3) Crater regions. Three best performing methods in each metric are color coded by rank, from lowest to highest RMS error $[1=$ green, $2=$ yellow, $3=$ red]. Uncolored cells indicate rank positions $>3 \mathrm{rd}$. Top performing methods: (1) UMD-CG. (2) PIXON and (3) GSFC-1GS. However, PIXON performed nearly as well with first differences only noted at the 7 th mean RMS decimal position.

An average of $80 \%$ of all map area is allocated to background and 20\% to craters, where each region is characterized as follows: 
Table 2

Method results: mean RMS error and method rank for 120 maps for $10^{\circ}$ estimates $80-90^{\circ}$ and method rank in set ( 1 green (best), 2 yellow, 3 red) (column 1 ). $10^{\circ}$ results and rank using Minkowski L2 metric (Duda et al., 2001) (column 2). Background only mean RMS error (column 3 ), in crater regions mean RMS error (column 3).

\begin{tabular}{|l|r|r|l|l|l|}
\hline Method I Metric & $\begin{array}{l}\text { 10-degree } \\
\text { MRMS }\end{array}$ & $\begin{array}{l}\text { 10-degree } \\
\text { L }^{2} \text { Norm }\end{array}$ & $\begin{array}{l}\text { Background } \\
\text { MRMS }\end{array}$ & $\begin{array}{l}\text { Crater } \\
\text { MRMS }\end{array}$ & $\begin{array}{l}\text { Category } \\
\text { Rank }\end{array}$ \\
\hline GSFC-IGS & 0.0211180 & 11.3412 & 0.0186679 & 0.0286845 & 1 \\
\hline GSFC-UM-RD & 0.0213130 & 11.4450 & 0.0190138 & 0.0284829 & 2 \\
\hline UMD CG & 0.0210508 & 11.3052 & 0.0178985 & 0.0302985 & 3 \\
\hline UMD Fourier & 0.0217578 & 11.6848 & 0.0183534 & 0.0316352 & \\
\hline PIXON & 0.0210573 & 11.3086 & 0.0175651 & 0.0310757 & \\
\hline
\end{tabular}

(2) Background: low amplitude spatial frequencies emulating the expected dynamic range and variation of lunar epithermal surface flux. Best performing methods identified (1) PIXON, (2) UMD-CG and (3) UMD-Fourier. Only small differences were noted between these results $<0.001 \mathrm{cnts} / \mathrm{sec}$, RMS. In background regions the dominant spatial frequency is near the direct current (DC) coefficient. Amplitudes of other higher frequency coefficients are comparatively small so, convergence to the DC baseline is expected for both IR methods and GSFC-IGS. As such, GSFC-IGS performed nearly as well as comparative restoration methods, in 4th place, with small first differences between GSFC-IGS and UMD-CG at the 4 th decimal place $[0.021118,0.0210508] \mathrm{cnts} / \mathrm{sec}$. (3) Craters: Table 2, column three defines results of evaluation of localized small spatial scale depressions in the epithermal neutron flux. Cratered regions are of primary interest for NASA's manned lunar exploration programs due to the potential for exploiting localized quantities of $(\mathrm{H})$ as a resource. These regions are inferred to occur primarily in regions of low illumination, but are defined here as cratered regions with variable sized depressions in the lunar surface epithermal neutron flux with variably high spatial frequencies at crater edges.

All method performances were worse than their (1) Global RMS errors, $>0.007$ RMS. Relative weighting of cratered and background results are consistent with the contributions factoring area-weights in the $10^{\circ}$ latitude band results. Higher RMS errors are seen due to the overlap of higher spatial frequencies in crater rims with noise frequencies at lower latitudes and are considered more difficult spatial frequencies to reconstruct. The ranges of method results indicate performance was consistent across the study, range $<0.003$ RMS. Best performers in this category were (1) GSFC-UM-RD, (2) GSFC-IGS and (3) UMD-CG. Importantly, with the exception of GSFC-UM-RD, results indicate that restoration methods did not improve significantly upon GSFC-IGS in cratered regions. Signal gains in cratered regions attributable to IR methods are of significant interest in this study due to the expected higher $\mathrm{H}$ concentrations and potentials for human exploration. Results in these higher spatial frequency regions did not identify significant gains vs, the IGS methodology. Additionally, UMD-CG and PIXON, use of a smoothing preprocessing step prior to the restoration step may have incurred additional unrecoverable signal losses in these regions.

\subsection{RMS error vs. latitude}

Fig. 4 and Table 3 illustrate the RMS error results as a function of latitude. Map SN increases as a function of higher latitude via the combined effects of the time, LRO mission's polar crossing trajectory, altitude and the expected overlapping of the LEND footprint over the poles. As a result the accumulated coverage is highest within $\sim 3^{\circ}$ of the pole where the greatest degree of

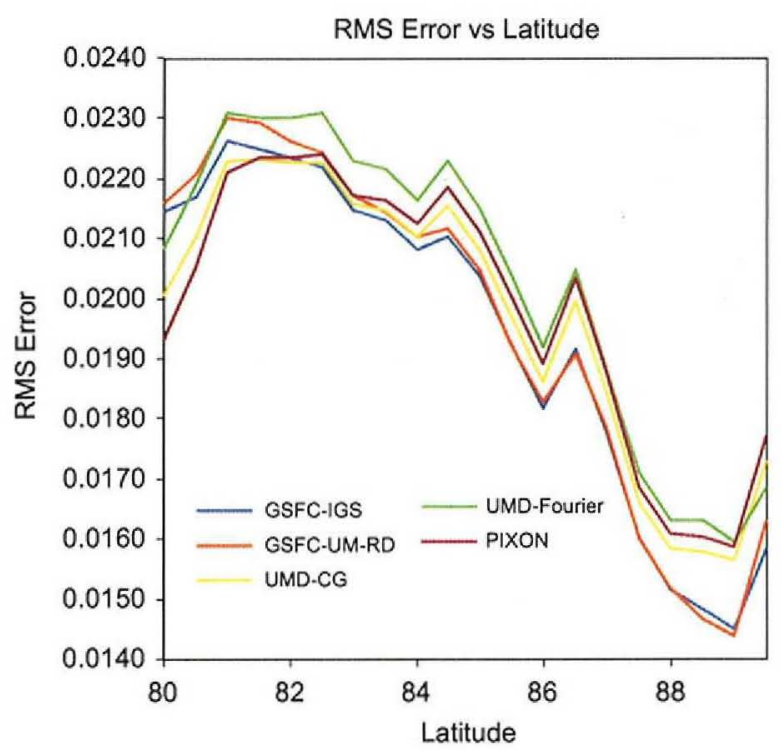

Fig. 4. Five IR methods latitude dependent mean RMS error averaged over 120 blind study images.

ground coverage overlap occurs. The accumulated coverage decreases significantly at latitudes below $\sim 87^{\circ}$, thereby decreasing the SN, Fig. 3a. To evaluate the impact of variable $\mathrm{SN}$ on method restorations, the map is decomposed as a function of 20 concentric latitude bands, $0.5^{\circ}$ in size ( 15 pixels wide) in which average RMS error is calculated, Eq. (1). A consequence of this binning of results is that each latitude band area decreases towards the pole yielding unequal weighting of bands in Fig. 4 .

Moving from low to high latitude (outside map to center), $80-82^{\circ}$ indicates PIXON and UMD-CG methods have superior reconstruction performances. This region constitutes $\sim 36 \%$ of the polar region evaluated. At $80^{\circ}$, images are dominated aerially by background regions with exceptions in some included craters in the randomized maps. As a result, due to lower spatial frequencies UMD-CG and PIXON performed best, which was consistent with both the overall and background results described in Table 2. As a fraction of area NP, SP craters are more prominent in the higher latitudes, which decreases the relative distribution of background to cratered pixels in these latitudes. Moving poleward to $81^{\circ}$, all performances are noted to improve, method performances intersect and RMS error decreases due to the higher SN approaching the poles. GSFC-UM-RD, GSFC-IGS results are equivalent, and alternate as the best performers. Trend discontinuities are indicated at $84.5^{\circ}$ and $86.5^{\circ}$, which are correlated with the generally higher occurrences of fixed craters for NP, SP maps. 
Table 3

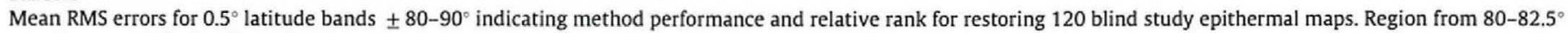
constitutes $\sim 50 \%$ of evaluated area.

\begin{tabular}{|c|c|c|c|c|c|c|c|}
\hline Latitude & GSFC-IGS & $\begin{array}{l}\text { GSFC-UM- } \\
\text { RD }\end{array}$ & U-MD-CG & $\begin{array}{l}\text { UMD } \\
\text { Fourier }\end{array}$ & PDXON & $\begin{array}{l}\text { Band Area } \\
\text { (fraction) }\end{array}$ & Rank \\
\hline 80 & 0.0214532 & 0.0215892 & 0.0200488 & 0.0208074 & 0.0192914 & 0.09741 & 1 \\
\hline 80.5 & 0.0216799 & 0.0220767 & 0.0210381 & 0.0219247 & 0.0205457 & 0.09251 & 2 \\
\hline 81 & 0.0226211 & 0.0230121 & 0.0223008 & 0.0230952 & 0.0220945 & 0.08753 & \\
\hline 81.5 & 0.0224759 & 0.0229242 & 0.0223110 & 0.0230088 & 0.0223411 & 0.08233 & \\
\hline 82 & 0.0223501 & 0.0226315 & 0.0222680 & 0.0230125 & 0.0223495 & 0.07746 & \\
\hline 82.5 & 0.0221881 & 0.0224350 & 0.0222824 & 0.0230964 & 0.0224117 & 0.07270 & \\
\hline 83 & 0.0214667 & 0.0217276 & 0.0215741 & 0.0222858 & 0.0217145 & 0.06750 & \\
\hline 83.5 & 0.0213115 & 0.0214573 & 0.0214711 & 0.0221484 & 0.0216352 & 0.06241 & \\
\hline 84 & 0.0208043 & 0.0210435 & 0.0210271 & 0.0216268 & 0.0212538 & 0.05756 & \\
\hline 84.5 & 0.0210452 & 0.0211569 & 0.0215559 & 0.0222956 & 0.0218656 & 0.05260 & \\
\hline 85 & 0.0203602 & 0.0204726 & 0.0208171 & 0.0214978 & 0.0211061 & 0.04755 & \\
\hline 85.5 & 0.0192660 & 0.0192408 & 0.0197538 & 0.0204231 & 0.0200792 & 0.04251 & \\
\hline 86 & 0.0181739 & 0.0182990 & 0.0186240 & 0.0191865 & 0.0189104 & 0.03734 & \\
\hline 86.5 & 0.0191531 & 0.0190952 & 0.0199612 & 0.0204816 & 0.0203486 & 0.03258 & \\
\hline 87 & 0.0177555 & 0.0178467 & 0.0184375 & 0.0188290 & 0.0187810 & 0.02751 & \\
\hline 87.5 & 0.0160259 & 0.0160105 & 0.0166219 & 0.0171157 & 0.0168565 & 0.02254 & \\
\hline 88 & 0.0151404 & 0.0151743 & 0.0158315 & 0.0163040 & 0.0160822 & 0.01741 & \\
\hline 88.5 & 0.0148233 & 0.0146576 & 0.0157896 & 0.0163187 & 0.0160251 & 0.01254 & \\
\hline 89 & 0.0145032 & 0.0143719 & 0.0156416 & 0.0159537 & 0.0158649 & 0.00751 & \\
\hline 89.5 & 0.0158328 & 0.0163019 & 0.0172973 & 0.0168458 & 0.0177089 & 0.00250 & \\
\hline
\end{tabular}

Error trends are elaborated in Table 3 where, the top two performers in each latitude band are highlighted $[1=$ green, $2=$ red $]$.

\subsection{Residuals}

Residual error distributions are derived by subtracting the true map from the restored map, $\mathbf{r}=\mathbf{g}^{\prime}-\mathbf{f}$. Histograms of $\mathbf{r}$ pixels provide the basis for metrics that evaluate the globalized IR reconstruction quality as well as the potential induction of map artifacts. Assuming residuals are normally distributed, a Gaussian fit of the $\mathbf{r}$ distribution provides both the mean $\mu_{r}$ and variance $\sigma_{r}^{2}$ terms. Binning of the residual errors was performed at $0.001 \mathrm{cps}$ intervals. As residual pixels are identical in weight and total numbers, ( $\sim 3.46 \times 10^{7}$ total pixels in the study), method residual error histograms have identical area. Direct comparisons of method residual distributions are performed as follows: (1) the residual error distribution mean $\mu_{r}$ deviation from 0 . quantifies the existence of bias in reconstructions. (2) Gaussian fit of the $\mathbf{r}$ distribution yields, amplitude and variance $\sigma_{r}^{2}$ terms which are inversely related. Optimally, for best IR performers the fit amplitude is increased and variance is decreased. (3) Artifact induction is inferred by greater numbers of high error pixels. To quantify this error for each method, the integral error in $\mathbf{r}$ histogram tails (a) outside $\pm>3 \sigma_{r}$ is derived. $\sigma_{r}$ from the
(PIXON) method (lowest variance) was used as the baseline, $\sigma_{\mathrm{PIXON}}=0.0163 \mathrm{cps}$.

Fig. 5 illustrates fits of each methods residual error distribution. Results of the bias study (1) indicated low bias for all methods $\leq 0.0022-0.2 \%$ of the mean detector count rate $0.88 \mathrm{cps}$. Best performing methods were (1) GSFC-UM-RD, (2) GSFC-IGS and (3) UMD-CG. The amplitude/variance metrics are coupled due to their mutual dependence. These results identified: (1) PIXON, (2) UMD-CG and (3) GSFC-IGS as having the highest amplitude/ lowest fit variance. Values for each methods residual error metrics are stated in Table 4.

Table 5 defines method performances in the analysis of residual tail areas. In each case the Tail $\mathrm{Hi}$ areas were approximately twice the areas of the Tail Lo areas. This is assumed due to Poisson statistics generating fewer 0 . cut-offs for pixel counts. Relative areas in these regions are approximately Tail Lo: $1.3 \%$ and Tail Hi: $2.4 \%$ of the total pixels processed for all methods. For normally distributed data, each tail above $\pm 3 \sigma$ should contain approximately $0.15 \%$ of all pixels processed indicating that in all methods tail areas are above the expected area and not normally distributed. Each methods tail areas are integrated above and below the two tail thresholds established using the PIXON baseline positions $\pm 3 \sigma_{\mathrm{PIXON}}$. For Tail Lo) (1) UMD-CG, (2) UMD-Fourier and (3) PIXON had the lowest tail areas. For Tail Hi) GSFC-UMD-RD had the lowest area followed by GSFC-IGS and UMD-CG. A third metric combining tail areas was established after noting the larger numbers of high intensity pixel 
Table 4

Moments from Gaussian fit of residual error. Mean indicates existence of bias as deviation from 0 . High amplitude and low sigma indicate higher number of pixels with low error. Rankings indicate all methods displayed low bias $<0.0023 \mathrm{cps}$, optimal GSFC-UM-RD. PIXON had highest amplitude and lowest variance followed by UMD-CG.

\begin{tabular}{|l|l|r|r|r|r|}
\hline $\begin{array}{l}\text { Residual Error } \\
\text { Analysis }\end{array}$ & Method & Amplitude & Mean & Sigma & $\begin{array}{l}\text { Category } \\
\text { Rank }\end{array}$ \\
\hline & GSFC-IGS & 748838 & -0.0015015 & 0.0176412 & 1 \\
\cline { 2 - 5 } GSFC-UM-RD & 735330 & -0.0009466 & 0.0180208 & 2 \\
\cline { 2 - 5 } & UMD CG & 775114 & -0.0017858 & 0.0169150 & 3 \\
\cline { 2 - 5 } & UMD Fourier & 738976 & -0.0022218 & 0.0178038 & \\
\cline { 2 - 5 } PIXON & 797804 & -0.0019891 & 0.0163151 & \\
\cline { 2 - 5 } & & & &
\end{tabular}

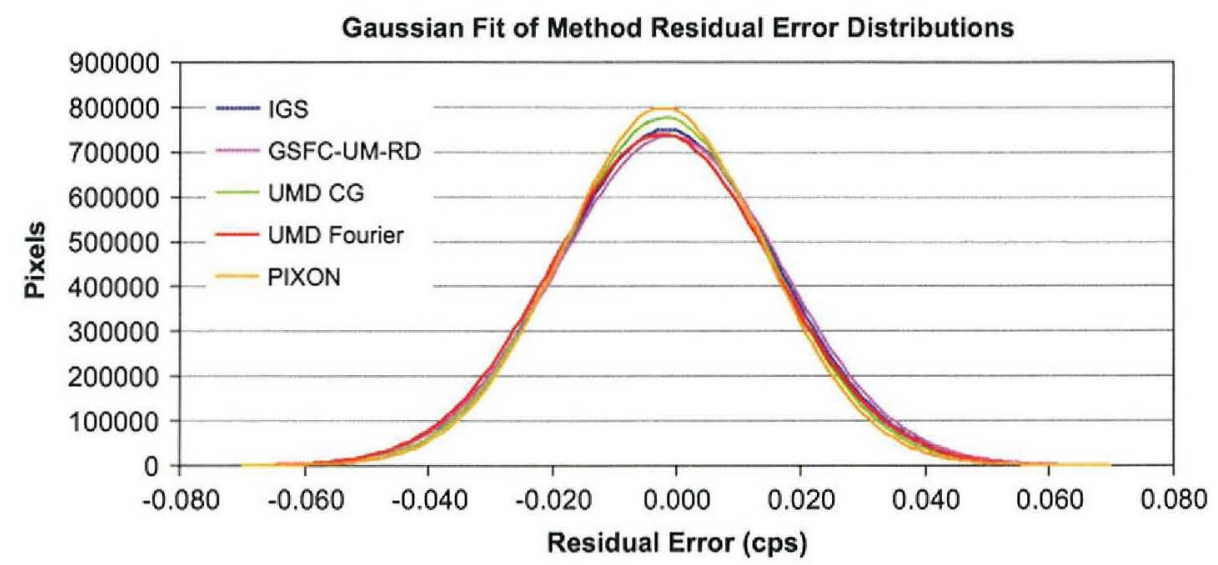

Fig. 5. Gaussian fits of method residual errors. Means indicate low bias for all methods. Variances indicate PIXON has lowest variance (also greatest peak amplitude), however examination of residuals in regions $\pm 3 \sigma$ indicate IGS smoothing has the lowest tail area in the high error region.

Table 5

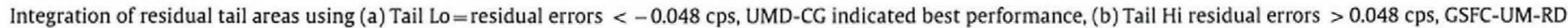
indicated best performance. Combined tail areas indicated IGS performed best.

\begin{tabular}{|c|c|c|c|c|c|}
\hline $\begin{array}{l}\text { Tail Error } \\
\text { Analysis }\end{array}$ & Method & $\begin{array}{l}\text { Tail Area } \\
\text { Lo }\end{array}$ & $\begin{array}{l}\text { Tails Area } \\
\text { Hi }\end{array}$ & $\begin{array}{l}\text { Total } \\
\text { Areas }\end{array}$ & $\begin{array}{l}\text { Category } \\
\text { Rank }\end{array}$ \\
\hline & GSFC-IGS & 431880 & 728209 & 1160089 & 1 \\
\hline & GSFC-UM-RD & 462744 & 717249 & 1179993 & 2 \\
\hline & UMD CG & 393927 & 802467 & 1196394 & 3 \\
\hline & UMD Fourier & 401568 & 900394 & 1301962 & \\
\hline & PIXON & 405688 & 844369 & 1250057 & \\
\hline
\end{tabular}

errors in the Tail Hi) metric. (3) Tail Totals yielded (1) GSFC-IGS, (2) GSFC-UM-RD and (3) UM-CG with the respectively lowest total tail area.

\section{Conclusions}

This paper described a two-phase blind study evaluation of four IR methods configured to evaluate and quantify restored lunar epithermal neutron flux maps. Background in orbital remote sensing of neutrons and LEND related factors was described with context in image restoration research. Comparison of IR methods was performed using a test database of synthetic LEND maps, derived from signals modeled from existing research and LEND instrument factors. IR methods were directly evaluated against a traditional smoothing process, GSFC-IGS to quantify the expected gains specific to IR deblurring vs. denoising. A 12-map training phase was included prior to the blind study to familiarize teams with the dynamics of LEND signals and facilitate method optimization. Teams optimized their IR methods on the training dataset via gridded search or simulated annealing methodology by evaluating method performance. GSFC-IGS was trained by establishing the optimal number of smoothing iterations (11) on the training set that minimized the RMS error.

The blind study phase comprised each teams reconstructing the 120 degraded map database using their respective training configurations.

Three types of IR method metrics were described (1) RMS error $\pm 80-90^{\circ}$, (2) RMS error vs. latitude and 3) residuals. In (1) only small gains were realized for UMD-CG and PIXON over 
GSFC EGS $<0.0001 \mathrm{cps}$ RMS. Relative improvements were approximately $2.4 \%$ of the expected LEND $0.88 \mathrm{cps}$ collimated count rate. It is inferred this is primarily due to the larger relative distributions of background vs. crater area, where background regions are more homogenous in intensity. However, for cratered regions of interest, GSFC-UM-RD, GSFC-IGS performed slightly better $\{0.02848,0.02868]$ cps than UMD-CG, PIXON [0.0303, $0.0311 \mathrm{l}$. (2) Map latitude analysis indicated method performance varied as a function of the relative distributions of background and cratered regions which varied with latitude. PIXON and UMDCG performed best in map outer latitudes characterized by lower SN and higher relative numbers of background pixels. GSFC-UMRD and GSFC-IGS performed better in map polar regions with higher $S N$ and higher relative numbers of cratered pixels, Fig. 4. This result may alternately reflect differences in training method optimization. (3) Residual error analysis via Gaussian fitting of residuals identified PIXON with the best performance at minimizing fit variance / maximizing amplitude [0.016, 797804]. However, the analysis of method tails indicates the GSFC-IGS method best minimized the existence of high error map pixels $> \pm 3 \sigma$ vs. reconstructions, $<0.04 \%$ tail area difference.

In summary, only small inconsistent signal gains in some metrics was realized by unbiased $\mathrm{R}$ methods over the default GSFC-IGS, specifically in the global metrics. Importantly, in more detailed regional analysis (craters) with higher "true" spatial frequencies, three of four IR methods performance lagged GSFCIGS in these critical signals. Similarly, all IR methods induced greater numbers of high error pixels (artifacts) vs. the default GSFC-IGS. Finally, IR methods were optimized with ground truth (generally not available in most applications) and yielded only small inconsistent gains vs. GSFC-IGS for the low LEND SN. These results would appear to limit the effectiveness of unbiased $I R$ methods in similar low SN applications. However, methods incorporating other physically based constraints into low SN IR methods may have potential in improving these results but may incur the risk of additional reconstruction errors due to the incorporation of new image modeling assumptions.

Future research will study the use and application of physically based modeling assumptions for LEND map reconstructions.

\section{References}

Anderson, L.E., Whitaker E. $A_{\text {, }}$ 1982. NASA catalogue of Lunar nomenclature Reference Publication 1097. National Aeronautics and Space Administration, Washington, DC, 20542, 183 pp.

Arnold, J., 1979. [Ce at the Junar polas regions. Journal of Geophysical Research 84 $5659-5668$

Boynton. W.V., Arnold, J., Barth, H., Brückner, ]., Drake, D., Englert, P.J., Evans, L,G., Feldman, W.C., Fellows, C., Fuller, K. Hamara, D, Harshman, K. Kerry. K. Litvak, M., Longmire, J., Metzger, A., Mitrofanov, ],G., Reedy, R.C., Shinohara, $C$ Squyres, S., Starr, R., Storms. S, Thornton, C. Ton'chev, A. Trombka, J., d'Uston C., Wänke, H., Turner, C., Ward, M., 2004. The Mars Odyssey gamma-ray spectrometer instrument suite. Space Science Reviews 110 (1-2), 37-83. doi:10.1023/B:SPAC.000002 1007,76126,15

Bussey, B., Lucey, P., Robinson, M. Spudts, P.D. Steutel, D., 2003. Permanent shadow in simple craters near the lunar poles. Geophysical Research Letters 30 (6), 1278. doi: $10.1029 / 2002 \mathrm{G} 2016380$.

Bussey, B., Spudis, P.D., 2004. The Clementine Atlas of the Moon. Cambridge University Press, Cambridge, UK, doi:10.2277/0521815282 $376 \mathrm{pp}$

Chin, G., Brylow, S., Foote, M., Garvin, J., Kasper, J., Keller, J., Litvak, M. Mitrofanov, I.G., Paige, D., Raney, K., Robiason, M., Sanin, A., Smith, D., Spence, H., Spudis, P.D. Stern, A. Zuber, M. 2007. Lurar reconnaissance orbiter overview: the jnstrument suite and mission. Space Science Reviews 129 (4), 391-419. doi: $10.1007 / \$ 11214-007-9153-y$.

Costa, L.F, Cesar, R.M., 2001. Shape Analysis and Classification: Theory and Practice. CRC Press, Boca Reton, FL, USA 658 pp.

Duda, R. Hart, P., Stork, D., 2001. Pattern Classification 2 nd edo. John Wiley and Sons, Danvers MA, USA $654 \mathrm{pp}$.

Eke, $V$, 2001. A speedy pixon image zeconstruction algorithm, Monthly Notices of the Royal Astronomical Society 324 (1) 108-118. doi:10.1046/\$1365-87112001. $04253 x$

Elphic, R.C., Bussey. B., Feld̈man, W.C., Lawrence, D.J., Maurice, S, Prettyman, T.M. Spudis, P.D. 2005. Using models of permanent stadow to constrain lunar polar water ice abundances, In: Proceedings of the 36 th Lunar and Planetary Science Conference, Clear Lake, Texas, USA, Abstract 2297.

Elphic, R.C., Eke, V.R., Teodoro. L.F.A, Lawrence, D. \&, Bussey D.8.J. 2007. Models of the distribution and abunciance of hydrogen at the lunar south pole. Geophysical Research Letters, 30 L13204, 5 pp, doi:10.1029/2007GLO29954.

Fetdmar, W.C., Barraclough, B.L., Binder, A.B., Elphic, R.C., Getenay, 1., Lawrence, D. J. Maurice, S., 1999a. Enhanced Hydrogen abundances near both lunar poles. 1n: Proceedings of the Workshop on New Views of the Moon2: Understanding the Moon through the Integration of Diverse Datasets 980 . Flagstaff, $A Z$, pp. 14-15.

Feldman, W.C., Barraclaugh, B., Binder, A.B., Flphic, R.C. Iawrence, D.J., 1998a. Fiuxes of fast epithermal neutrons from Lunar Prospector; evidence for ice at the lunar poles. Science 281 (5382), 1496-1500. doi:10.1126/scierce.281.5382.1496.

Feldman, W.C., Barzaclaugh, B.L... Binder, A.B., Elphic, R.C. Lawrence, D.l. Maurice, S. 1998b. Major compositional units of the Moon: fluxes of fast and thermal neutrons, Science 281 (5382), 1489-1493. doj:10.1126/science.281.5382.1489.

Feldman, W.C, Baraclough, B.L, Binder, A,B., Elphic, R.C., Lawrence, D.J., Maurice, $S, 2000$. Polar hydrogen deposits on the Moon. Journal of Geophysical Restearch 105 (E2), 4175-4195.

Feldman, W.C., Barraclough, B.L.. Binder, A.B., Futler, K.R., Lawrence, D.J., Maurice, S. Millef, M.C., Prettyman, T.H., 1999b. The Lunar Prospector gamma-ray and neutron spectrometers. Nuclear lnstruments and Methods in Jhysics Research Section A: Accelerators, Spectrometers, Detectors and Associated Equipment $422(\mathrm{~T}-3), 562-566$.

Feldman, W.C., Boynton, W, $V_{,}$Drake, D., 1993. Planetary neutron spectroscopy from orbit. In: Pieters, C.M., Englert, P.A.J. (Eds), Remote Geochemical Analysis: Elemental and Mineralogica] Composition. Cambridge University Press, Cambridge, UK, pp. 213-234.

Gonzalez, R.C., Woods, RE, 1993. Digital Image Processing. Addison-Wesley Publishing Co., Reading, MA $716 \mathrm{pp}$.

Ivatury, V., McClanahan, F.P., 2009. Image restoration of Lunar neutron albedo maps for the Lunar Exploration Neutron Detector (LEND). In: Proceedings of the 40th Lunar and Planetary Science Conference, Woodlands, TX, USA, ID1 134

Koenderink, 3.J., 1984. The structure of images, Biological Cybernerics 50 (5), 363-370. doi:10.1007/BF00336961.

Lawrence, D.J., Elphic, R.C., Feldman, W.C., Hagercy, J., Puerter, R., Prettyman, $T$. 2006. Improved modeling of Lunar Prospector neutron spectrometer data: implications for hydrogen deposits at the Lunar poles. Journal of Geophysical Research 111, E08001, doi:10.1029/2005/E002637.

McClanahan, T.P., Evans, LG, Finch, M.J., Mitrofanov, [.G., Starr, R., 2007. Application of image restoration (jansson-Van Cittert) to planetary remote sensing albedo maps. In: Proceedings of the 39th Lunar and Planetary Science Conference (LPSC), Woodlands, TX, USA, ID2408.

McClanahan, T.P., Evans, L.G. Mitrofanov, I.G., Starr, R.D., 2009. Fast ray tracing of Lunar digital elevation models. In: Proceedings of the 40th Lunar and Planetary Science Conference, Woodlands, TX, USA, ID2092.

Mitrofanov, J.G. Anfmov, D., Boynton, W.V. Hamara, D., Kozyrev, A., Krylov, A. Litvak, M., Saunders, R., Santn, A, Shinohara, C., Shvetsov, V., Tretyakov, V., 2002. Maps of subsurface hydrogen from the high energy neutron detector (HEND). Mars Odyssey. Science 297 (5578) 78-81, doi:10.1126/science 1073616.

Mitrofanov, l.G., Bartels, A., Bobrovnitsky, Y.l., Boynton, W.V., Chin, G., Enos, H., Evans, L.G., Floyd, S.F, Garvin, ]., Golovin, D.V., Grebennikov, A.S., Harshman, K., Kazakov, L.L., Keller, J., Konovalov, A.A., Kozyrev, A.S., Krylov, A.R., Litvak, M.L., Malakhov, A.V., McClanahan, T.P., Milikh, G.M., Mokrousov, M.I., Ponomareva, S., Sagdeev, R.Z., Sanin, A.B., Shevchenko, V.V., Shvetsov, V.N., Starr, R, Timoshenko, G.N., Tomilina, T.M., Tretyakov, V.1., Trombka, J., Troshin, V.S." Uvarov, V.N. Varennikov, A.B., Vostrukhin, A.A. 2009. Lunar exploration neutron detector fos the NASA Lunar Reconnaissance Orbiter. Space Science Reviews 150 (1-4), 183-207, doi: 10.1007/s11214-009-9608-4.

Mitrofanov. I.G., Bartels, A., Bobrovitsky. Y.L, Boynton, W.V., Chin, G., Evans, L.G., Garvin, J., Golovin, D.V., Grebennikov, A., Harshman, K., Kazakov, L., Keller, ]., Konovalov, A.A, Kozyrev, A.S., Krylov, A., Litvak, M., Malakhov, A.V., McClanahan, T.P., Milikh, G., Mokrousov, M.L, Sagdeev, R.Z., Sanin, A., Shevchenko, $V$., Shvetsov, $V$, Starr, R., Timoshenko, G.N. Tomilina, T.M., Tretyakov, V.1. Trombka, \}. Troshin, V., Uvarov, V. Varenikov, A, Vostrukhin, A., 2008. Experiment LEND for the NASA Lunar Reconnaissance Orbiter. Astrobiology 8 (4), 793-804. doi:10.1089/ast,2007,0158.

Mitrofanov, l.G. Boynton, WV. Chin, G, Evans, L.G. Garvin, I. Golovin, D.V., Harsliman, K., Kozyrev, A., Litvak, M., Malakhov, A.V.. McClanahan, T.P., Milikh, G., Mokrousov, M.L., Nandikotkur, G., Nuzhdin, $i_{\text {. }}$ Sagdeev, RZ., Sanin. A. Shevchenko, V., Shvetsov, V., Starr, R., Timoshenko, G.N., Tomilina, T.M., Tretyakov, V.l., Trombka, l., Troshin, V., Uvarov, V., Varenikov, A., Vostrukhin, A. 2010. LEND Experiment onboard LRO: testing local areas with thigh concentrations of hydrogen at the lunar poles. In: Praceedings of the 41 st Lunar and Planetary Science Conference, Woodlands, TX, USA, ID2250.

Nesbitt, L.A., 2004. Clinical Research: What It Is And How It Works. Jones and Bartletr Publishers, Sudbury, MA, USA 274 pp.

Ngtiyen, $N$., Golub, G., Milasfar, P., 2001. A computationally efficient super. resolution image reconstruction algorithm. Institute of Electronic and Electrical Engineers: Transactions on Image Processing 10 (N4), 573-583.

Neubert, J., Lucey, P., Taylor, J.. 2005. Properties of permanertly shadowed regolith. 2005, in: Proceedings of the 36th Lunar and Planetary Scjence Conference, Woodtands, JX, USA, ID1613.

Pelowitz, D., 2008. MCNPX Users Manual: Version 2.6, US Government Export Controlled, Los Alamos National Laboratory report, LA-CP-07-1473, DE-AC5206NA25396, $551 \mathrm{pp}$. 
Perona, P., Malik, J., 1987. Scale space edge detection using anisotropic diffusion. International Electronics and Engineering Transactions on Pattern Analysis and Machine Intelligence 12 (7), 629-639. doi:10.1109/ 34.56205 .

Peutrer, R., Gosnell, $T$, Yahill, A., 2005. Digital image reconstruction: deblurring and denoising. Annual Reviews of Astronomy and Astrophysics 43, 139-194. doj:10.1146/anmurevastro.43.112904.104850.

Press, W.H. Flannery, B.P., Teukoisky, S.A., Vellerling, W.T, 2008, Numerical Recipes in C3rd edn. Cambridge University Press, New York, NY, USA. doj:10.1137/1031025.

Rosenfeld, A., Kak, A., 1982. Digital Picture Processing 2nd edn. Academic Press, Orlando. Florida USA 349 pp.

Saał, A.E., Cascio, M.L, Cooper, R.F., Hauri, E.H.. Rutherford, M.C., Van Orman, JA. 2008. Volatile content of lunar volcanic glasses and the presence of water in the Moon's interios. Nature $454,192-195$ doi:10.1038/ nature07047.

Ugbeme, O.A., Saber, E., Wu, W., 2007. An automated aigorithm for the identification of artifacts an monted and nolsy images, Joturnal of Electronic Imaging 16 (3. doi: $10.1117 / 12.773053$ Article \#033015.

Vasavada, A, Paige, D, Woad, \$, 1999, Near surface temperatures on Mercury and the Moon and the stability of polar ice deposits. [carus 141 (2), 179-193. doi: $10.1006 /$ icar.1999.6175.

Vondrak, $R$, Stider, D, 2003. Ice at the Lunar poles. American Scientist 91, 322-329.

Weiner, N., 1942. Excrapolation, Interpolation and Smoothing of Stationary Time SeriesmiT Press, Cambridge, MA, USA 163 pp.

Wolpert. D.H., Macready, W.C., 1997. No free lunch theorems for optimization. Institute for Electronics and Electrical Engineers: Transactions on Evolutionary Computation 1(1), 67-82. doi:10.1109/4235.585893. 